\title{
Influencia del género y del sexo en las actitudes sexuales de estudiantes universitarios españoles
}

\section{Influence of the sex and gender in the sexual attitudes in Spanish university students}

\author{
Elisa Larrañaga ${ }^{1} \quad$ Santiago Yubero Marta Yubero \\ Universidad de Castilla-La Mancha, España
}

(Rec: septiembre de 2012 - Acep: noviembre de 2012)

\begin{abstract}
Resumen
Los jóvenes son un grupo de especial importancia en la prevención de la salud sexual, lo que lleva a que resulte de interés el análisis de los factores que determinan la conducta sexual. El propósito de este trabajo fue estudiar la relación entre el género y la sexualidad. Concretamente, el objetivo ha sido analizar la relación entre el machismo y los mitos románticos con las actitudes sexuales. Se ha empleado un cuestionario autoadministrado que consta de tres escalas, Double Standard Scale, Escala de Mitos Románticos y Escala de Actitudes Sexuales, a 262 estudiantes de la Universidad de Castilla-La Mancha (España). Los datos fueron recogidos durante el curso 2011-2012. Hemos aplicado análisis univariantes para conocer las relaciones entre las variables. Los resultados indican que las actitudes sexuales están vinculadas con el machismo y los mitos románticos. En futuros programas de intervención y de educación sexual es necesario considerar la variable de género.

Palabras clave: género, actitudes sexuales, machismo, mitos románticos, jóvenes y salud sexual.
\end{abstract}

\begin{abstract}
The young people is a group of special importance for the prevention of health sexuality, reason why it turns out from interest to study the determinants of sexual behavior. The purpose of this study was to examine the link between gender and sexuality. The objective was to analyze the machism and romantic myths associated with sexual attitudes. For the survey, a self-administered paper based questionnaire was used. The Double Standard Scale, Romantic Myths Scale and Sexual Attitudes Scale were administered to a sample of 262 university students of University of Castilla-La Mancha (Spain). Data were collected between 2011 and 2012. Were used univariant model were estimated to relation about factors. The results of this study indicate that sexual attitudes are relation with machism and romantics myths. The gender should be taken into consideration in planning prevention programmes and sexual education.
\end{abstract}

Keywords: gender, sexual attitudes, machism, romantics myths, young people and sexual health.

\footnotetext{
1 Correspondencia a: Universidad de Castilla-La Mancha, Facultad de Trabajo Social, Departamento de Psicología. Edificio Melchor Cano, Campus Universitario s/n, 16071 Cuenca (España).E-mail: elisa.larranaga@uclm.es.
} 


\section{Introducción}

Si tenemos en cuenta que para la gran mayoría la sexualidad es un elemento fundamental en la vida y necesario para el equilibrio personal (87\% de los hombres y $78 \%$ de las mujeres, según la Encuesta Nacional de Salud Sexual del Ministerio de Sanidad y Política Social de España, 2009), garantizar una sexualidad segura se convierte en un objetivo social básico. Ha de entenderse la sexualidad como un estado de bienestar físico, emocional y mental, que requiere la posibilidad de tener experiencias sexuales seguras (Organización Mundial de la Salud, 2006). Incurrir en un comportamiento sexual de riesgo conlleva consecuencias a corto y largo plazo; entre ellas, embarazos no deseados, interrupciones voluntarias del embarazo y el contagio de enfermedades de transmisión sexual.

Los jóvenes se considera un colectivo susceptible de incurrir en la práctica de relaciones sexuales de riesgo, ya que tienden a no utilizar métodos anticonceptivos eficaces en sus relaciones, tienen un inicio precoz en sus relaciones coitales y un mayor número de parejas ocasionales (Muñoz-Silva, Sánchez-García, Martins \& Nunes, 2009; Paz, Castro, Madrid \& Buela-Casal, 2010; Teva, Paz \& Buela-Casal, 2009). Navarro, Carrasco, Sánchez y Torrico (2004) atribuyen este comportamiento sexual de riesgo en jóvenes a diversos factores, entre ellos tener una actitud negativa frente al uso de anticonceptivos. Teva et al. (2011) han constatado que un $37 \%$ de los jóvenes encuestados afirman que el uso de anticonceptivos resta placer a las relaciones sexuales, que usar métodos anticonceptivos quita espontaneidad a las relaciones (38\%) y que el preservativo es complicado de usar (62\%). Una actitud desfavorable hacia el uso de métodos anticonceptivos lleva a una mayor probabilidad de rechazar su utilización e incurrir en un comportamiento sexual de riesgo.

Es importante resaltar las diferencias observadas en el comportamiento sexual entre hombres y mujeres en distintas investigaciones. Los hombres tienen un inicio más precoz de las relaciones sexuales, mayor número de parejas y más parejas ocasionales; las mujeres tienen relaciones sexuales básicamente con su pareja, en el marco de una relación estable y asociada con el amor (Failde, Lameiras \& Bimbela, 2008; Petersen \& Hyde, 2010; Muñoz-Silva et al., 2009; Puente et al., 2011; Teva et al., 2009). García-Vega, Menéndez, García y Rico (2010) explican estas diferencias del comportamiento sexual en relación con la identidad de género.

Por otra parte, aunque las mujeres tienen mayor conciencia del peligro, su comportamiento no se traduce en acciones reales, no encontrando diferencias en el empleo de preservativos ni en la adopción de medidas de control de natalidad (Calafat et al., 2009), especialmente con sus parejas estables (Muñoz-Silva et al., 2009). En la sexualidad, más que el conocimiento parece que es la actitud la que actúa como predictor del comportamiento sexual (Yubero, Yubero \& Larrañaga, en prensa).

Las actitudes hacia la sexualidad humana han estado siempre relacionadas con la moral imperante en la sociedad de la época (Sierra, Rojas, Ortega \& Marín, 2007). Tradicionalmente, determinados comportamientos sexuales eran valorados de diferente modo según fueran realizados por hombres o por mujeres (Milhausen \& Herold, 1999). Esta distinta valoración de ciertas conductas sexuales es conocida como doble moral sexual. La doble moral asume una mayor libertad para el hombre que para la mujer en las relaciones sexuales, la existencia de múltiples parejas, el inicio sexual a edades más tempranas y relaciones sexuales exentas de compromiso (Sierra et al., 2007).

Los valores ante la sexualidad han cambiado en nuestro entorno sociocultural (Giddens, 1995; Chaplin, 2000). El Informe de la Juventud en España (INJUVE, 2008) señala que los jóvenes valoran la sexualidad y la definen como una experiencia placentera y saludable. Además, en los últimos años hemos sido testigos de múltiples cambios en la sociedad dirigidos a la consecución de modelos más igualitarios entre hombres y mujeres. Sin embargo, algunos autores siguen evidenciando la existencia de creencias machistas que llevan a actitudes sexuales más restrictivas para las mujeres que para los hombres (Diéguez, Sueiro \& López, 2003; de Lemus, Moya \& Glick, 2010), en las que impera la doble moral (Milhausen \& Herold, 1999) y el machismo sexual (Díaz, Rosas \& González, 2010).

Castañeda (2002) define el machismo como el conjunto de creencias, actitudes y conductas que ponen de manifiesto la superioridad del hombre sobre la mujer. Se trata de un concepto similar al sexismo clásico. La manifestación del machismo son comportamientos relacionados con la educación social tradicional de los hombres, que los sitúa en una posición de ventaja al reducir la libertad de decisión de la mujer. En estudios previos (Pleck, Sonenstein \& Ku, 1993) se ha mostrado que una actitud sexista puede llevar al incremento de los embarazos no deseados al vincularse con actitudes relacionadas con un menor empleo del preservativo y su uso inconsistente. Caron, Davis, Halteman y Stickle (1993) encontraron que los jóvenes con actitudes machistas (medidas a través de una escala de doble 
moral) mostraban actitudes más negativas hacia el preservativo, manifestándose menos dispuestos al uso de condones durante sus relaciones sexuales.

El amor romántico también favorece las relaciones de poder en la pareja, llevando a la entrega total de la mujer y al dominio del hombre (Selva, 2011). De hecho, como afirma Medrano (2012), los mitos románticos son una expresión de la sociedad patriarcal que justifican el machismo. El machismo es la forma de dominio, el romanticismo la respuesta de sumisión (Moreno, 2003), contribuyendo al mantenimiento del sexismo (de Lemus et al., 2010). Desde principios del siglo XIX existe una conexión entre la sexualidad y el amor romántico. En la cultura occidental se encuentra una relación estrecha entre ambos conceptos, llevando a considerarse que estar enamorado es la base fundamental para establecer una pareja y permanecer en ella (Ubillos, et. al, 2001). Con este concepto se incide en las emociones, los afectos y el cuidado de las relaciones interpersonales como parte del rol de la mujer. Ferrer, et al. (2008a) informan que más del $80 \%$ de la población española acepta el estilo de amor romántico, tanto varones como mujeres.

Los mitos románticos son creencias sobre el amor, que con una gran carga afectiva son compartidos socialmente. Expresan verdades absolutas y poco flexibles que son ficticias, absurdas, engañosas, irracionales e imposibles de cumplir (Yela, 2003), y muy resistentes al cambio y al razonamiento (Bosch \& Ferrer, 2002). Diversas investigaciones (Barrón, Martínez-Iñigo, de Paul \& Yela, 1999; Esteban \& Távora, 2008; Ferrer, Bosch \& Navarro, 2010) han demostrado que los mitos románticos son aceptados por un elevado porcentaje de personas en nuestro país. Se encuentra más aceptación en las mujeres, en las personas mayores y en las personas con menos formación académica.

Yela (2003) realiza una revisión de los principales mitos románticos. Por el interés para nuestro estudio destacamos seis. Mito de la 'media naranja' es la creencia de que tenemos la pareja predestinada y que es la única elección posible. La aceptación de este mito lleva a una relación con los límites de exigencia deteriorados (muy exigentes o muy laxos) por la presión de que todo vaya bien. Mito del emparejamiento es la creencia absoluta en la monogamia amorosa. Mito de la omnipotencia es la creencia de que el amor lo puede todo, el amor es suficiente para superar y resolver todos los conflictos. Este mito puede ser empleado como una excusa para aceptar todos los comportamientos. Mito del matrimonio, creencia de que el amor conduce a una pareja estable y que la satisfacción sexual debe darse en el matrimonio/pareja. Este mito une la pasión con la permanencia, por lo que es muy fácil que lleve a la decepción. Mito de la pasión eterna es la perdurabilidad del amor tras años de convivencia. Mito de los celos es la creencia de que los celos son un signo de amor. Los mitos románticos constituyen una garantía de exclusividad y de fidelidad (sólo se puede amar a una persona).

Basándonos en los datos aportados por la investigación previa, nos proponemos analizar la relación entre el género y la sexualidad, centrándonos en la influencia del machismo y de los mitos románticos sobre las actitudes sexuales de los jóvenes universitarios. Pensamos que la perspectiva de género podría resultar interesante para abordar los programas de educación sexual.

\section{Metodología}

\section{Participantes}

La muestra está compuesta por 262 jóvenes estudiantes universitarios de los grados de Trabajo Social y Educación Social, del campus de Cuenca de la Universidad de Castilla-La Mancha. La distribución por sexos se encuentra sesgada (77.8\% mujeres y $21.5 \%$ hombres) en función de la constitución de origen. La media de edad es de 20.92 años (hombres 25.87 años y mujeres 20.60 años).

\section{Instrumento}

Se optó por un cuestionario autoadministrado para favorecer la sensación de intimidad y confidencialidad.

Para medir la actitud hacia las relaciones sexuales y el uso de métodos anticonceptivos, hemos empleado la Escala de Actitud Sexual (Yubero et al., en prensa). Consta de 15 ítems referentes a la actitud hacia las relaciones sexuales completas (con penetración), las ventajas o desventajas percibidas en el uso de anticonceptivos y la disposición a usarlos. El sujeto debía expresar su grado de acuerdo o desacuerdo a través de una escala Likert de cinco puntos (1-Totalmente en desacuerdo, 5-Totalmente de acuerdo).

Para la medida del machismo se ha empleado la Double Standard Scale (DSS; Caron et al, 1993), que ha sido adaptada en nuestro país por Sierra et al. (2007). Está formada por 10 ítems que se contestan en escala tipo Likert de 5 puntos desde 1 (totalmente en 
desacuerdo) hasta 5 (totalmente de acuerdo). Permite evaluar la doble moral en el área de la sexualidad. La consistencia interna de la escala, medida con el alfa de Cronbach, es de 0.60 .

Los mitos románticos se han medido aplicando la escala de Ferrer et al. (2010). Consta de 10 ítems que describen los mitos evaluados: media naranja, pasión eterna, omnipotencia, matrimonio, emparejamiento y celos. A los participantes se les pidió que mostraran su grado de acuerdo o desacuerdo con el contenido del ítem en una escala de 5 puntos (donde 1 indicaba completo desacuerdo y 5 completo acuerdo). El coeficiente alpha de Cronbach para el conjunto de los ítems fue de .52 , similar al encontrado por los autores.

\section{Procedimiento}

Los sujetos participaron libremente de manera voluntaria e individual, habiendo sido previamente informados del objetivo de la investigación. El cuestionario fue aplicado en el curso 2011-2012, dentro de las aulas de cada una de las facultades, con el consentimiento previo de los profesores de la titulación que ocupaban el espacio en el momento de completarlo.

\section{Análisis de datos}

En primer lugar se ha realizado un análisis descriptivo de las variables de estudio: actitud sexual, machismo y mitos románticos. Posteriormente se han realizado análisis univariantes sobre los factores de la actitud sexual.

Los análisis estadísticos han sido desarrollados a través del paquete informático SPSS/PC (versión 19).

\section{Resultados}

Los estudiantes universitarios muestran su mayor acuerdo con la actividad sexual activa y la anticoncepción (hombres: $89.3 \%$; mujeres: $87.7 \% ; \chi 2=0.11, p=$ .476). Aunque los hombres rechazan en mayor medida el empleo de medidas anticonceptivas (hombres: 10.2\%; mujeres: $2.6 \% ; \chi 2=5.82, p<.030$ ) y muestran un rechazo personal más elevado hacia el empleo de los preservativos (hombres: $51.1 \%$; mujeres: $32.3 \% ; \chi^{2}=5.55, p<$ .015). Las mujeres consideran más importante la estabilidad de la pareja para mantener relaciones sexuales (hombres: $67.6 \%$; mujeres: $81.8 \% ; \chi 2=3.65, p<.050$ ).

Los análisis indicaron la existencia de diferencias significativas en la aceptación del machismo en función del género. El 20.8\% de los hombres mostraron su acuerdo con las creencias machistas, frente al 9.8\% $(\chi 2=4.61, p<.032)$ de las mujeres. Las medias reflejan las diferencias entre hombres y mujeres (hombres $M=1.67$; mujeres $\left.M=1.97 ; t_{235}=2.86 ; p<.005\right)$. Las diferencias se encuentran en que los hombres mantienen su rol de poder en las relaciones sexuales: tener múltiples encuentros para ganar experiencia (hombres $36.4 \%$, mujeres $10 \%, \chi 2=30.10, p<.000)$ y tomar el rol dominante en la relación sexual (hombres $18 \%$, mujeres $7 \%, \chi 2=11.74, p<.019)$.

El romanticismo está presente en un elevado porcentaje de hombres (78.3\%) y de mujeres (81.9\%), no encontrándose diferencia significativa según sexo $(\chi 2=0.31, p=.358)$. Los jóvenes universitarios creen mayoritariamente en la pasión eterna (87.3\%), en la omnipotencia del amor (85.4\%) y en el mito de la media naranja (85\%). Más de la mitad (61\%) cree en el mito del matrimonio, un $30 \%$ en el mito del emparejamiento y casi un $22 \%$ cree que los celos son una prueba de amor.

\section{Género, sexo y actitudes sexuales}

Los modelos de género no alcanzan significación estadística para la estabilidad de la pareja ni para la aceptación de la anticoncepción. En el factor de rechazo de los métodos anticonceptivos, las dos variables de género y sus interacciones son significativas, también la interacción con el sexo, aunque la variable sexo no llega a alcanzar significación. El rechazo social del preservativo es significativamente superior en los/las jóvenes machistas y en los/las jóvenes románticos/as. El rechazo personal del preservativo es superior en los hombres y en los/las jóvenes machistas. En este factor alcanza significación la interacción del romanticismo y el sexo. El análisis de medias de los grupos de estudio indica que en el nivel alto de romanticismo se equiparan las mujeres $(M=2.73)$ a los hombres $(M=2.76)$ en el rechazo personal de los preservativos. Los resultados pueden observarse en la Tabla 1.

\section{Discusión}

En España, el Ministerio de Sanidad y Política Social, dentro de las acciones previstas en su Plan de Calidad para el Sistema Nacional de Salud, elaboró en 2009 la primera Encuesta Nacional sobre Salud Sexual. El objetivo fue conocer las necesidades de hombres y mujeres de todas las edades para planificar 
UNIVERSITARIOS ESPAÑOLES

Tabla 1.

Actitud sexual. Diferencias por sexo y por género

\begin{tabular}{|c|c|c|c|c|c|c|c|}
\hline Fuente & & $M$ & $D T$ & $F$ & $p$ & $\eta$ & $\mathbf{R}^{2}$ \\
\hline \multicolumn{8}{|l|}{ Factor I: Estabilidad de la pareja } \\
\hline Modelo & & & & 1.02 & .503 & .838 & .016 \\
\hline Sexo & Hombre & 2.67 & 0.83 & 1.89 & .181 & .070 & \\
\hline Machismo & Mujer & 2.99 & 0.68 & 1.24 & .297 & .533 & \\
\hline \multirow[t]{5}{*}{ Romanticismo } & Bajo & 2.95 & 0.70 & 0.92 & .581 & .458 & \\
\hline & Medio & 2.91 & 0.73 & & & & \\
\hline & Bajo & 2.78 & 1.26 & & & & \\
\hline & Medio & 2.91 & 0.68 & & & & \\
\hline & Alto & 3.08 & 0.77 & & & & \\
\hline Sexo*Machismo & & & & 0.18 & .839 & .014 & \\
\hline Sexo*Romanticismo & & & & 0.50 & .504 & .053 & \\
\hline Machismo*Romanticismo & & & & 0.72 & .723 & .646 & \\
\hline Sexo*Machismo*Romanticismo & & & & 3.60 & .059 & .019 & \\
\hline \multicolumn{8}{|c|}{ Factor II: Rechazo de los métodos anticonceptivos } \\
\hline Modelo & & & & 3.45 & .000 & .918 & .652 \\
\hline Sexo & Hombre & 1.61 & 0.73 & 0.93 & .340 & .020 & \\
\hline Machismo & Mujer & 1.36 & 0.53 & 3.06 & .001 & .610 & \\
\hline \multirow[t]{5}{*}{ Romanticismo } & Bajo & 1.33 & 0.61 & 2.95 & .001 & .621 & \\
\hline & Medio & 1.57 & 0.51 & & & & \\
\hline & Bajo & 1.14 & 0.22 & & & & \\
\hline & Medio & 1.41 & 0.58 & & & & \\
\hline & Alto & 1.50 & 0.74 & & & & \\
\hline Sexo*Machismo & & & & 9.66 & .000 & .518 & \\
\hline Sexo*Romanticismo & & & & 7.10 & .000 & .441 & \\
\hline Machismo*Romanticismo & & & & 3.45 & .000 & .837 & \\
\hline Sexo*Machismo*Romanticismo & & & & 5.08 & .007 & .490 & \\
\hline \multicolumn{8}{|c|}{ Factor III: Rechazo social del preservativo } \\
\hline Modelo & & & & 2.02 & .003 & .867 & .438 \\
\hline Sexo & Hombre & 1.64 & 1.07 & 1.28 & .263 & .026 & \\
\hline Machismo & Mujer & 1.49 & 0.54 & 3.20 & .000 & .605 & \\
\hline \multirow[t]{5}{*}{ Romanticismo } & Bajo & 1.38 & 0.48 & 2.12 & .013 & .515 & \\
\hline & Medio & 1.79 & 0.95 & & & & \\
\hline & Bajo & 1.33 & 0.49 & & & & \\
\hline & Medio & 1.53 & 0.73 & & & & \\
\hline & Alto & 1.52 & 0.54 & & & & \\
\hline Sexo*Machismo & & & & 0.70 & .646 & .081 & \\
\hline Sexo*Romanticismo & & & & 0.37 & .894 & .044 & \\
\hline Machismo*Romanticismo & & & & 1.53 & .060 & .696 & \\
\hline Sexo*Machismo*Romanticismo & & & & 0.58 & .561 & .005 & \\
\hline
\end{tabular}




\begin{tabular}{|c|c|c|c|c|c|c|c|}
\hline \multicolumn{8}{|c|}{ Factor IV: Rechazo personal del preservativo } \\
\hline Modelo & & & & 1.82 & .017 & .867 & .391 \\
\hline Sexo & Hombre & 2.55 & 0.78 & 6.73 & .013 & .150 & \\
\hline Machismo & Mujer & 2.27 & 0.77 & 2.38 & .009 & .580 & \\
\hline \multirow[t]{5}{*}{ Romanticismo } & Bajo & 2.23 & 0.74 & 1.50 & .130 & .476 & \\
\hline & Medio & 2.57 & 0.81 & & & & \\
\hline & Bajo & 2.36 & 0.84 & & & & \\
\hline & Medio & 2.27 & 0.73 & & & & \\
\hline & Alto & 2.74 & 0.90 & & & & \\
\hline Sexo*Machismo & & & & 2.48 & .060 & .207 & \\
\hline Sexo*Romanticismo & & & & 2.85 & .037 & .231 & \\
\hline Machismo*Romanticismo & & & & 1.50 & .091 & .697 & \\
\hline Sexo*Machismo*Romanticismo & & & & 0.01 & .985 & .000 & \\
\hline \multicolumn{8}{|c|}{ Factor V: Aceptación de la anticoncepción } \\
\hline Modelo & & & & 0.98 & .537 & .759 & .009 \\
\hline Sexo & Hombre & 3.37 & 0.81 & 0.61 & .439 & .012 & \\
\hline Machismo & Mujer & 3.37 & 0.75 & 0.95 & .545 & .317 & \\
\hline \multirow[t]{5}{*}{ Romanticismo } & Bajo & 3.41 & 0.79 & 0.97 & .512 & .333 & \\
\hline & Medio & 3.29 & 0.72 & & & & \\
\hline & Bajo & 3.12 & 0.87 & & & & \\
\hline & Medio & 3.39 & 0.75 & & & & \\
\hline & Alto & 3.35 & 0.88 & & & & \\
\hline Sexo*Machismo & & & & 1.66 & .151 & .169 & \\
\hline Sexo*Romanticismo & & & & 1.72 & .136 & .174 & \\
\hline Machismo*Romanticismo & & & & 1.03 & .502 & .596 & \\
\hline Sexo*Machismo*Romanticismo & & & & 2.91 & .056 & .023 & \\
\hline
\end{tabular}

una atención sexual adecuada. En marzo del año siguiente se aprobó la Ley Orgánica 2/2010 de salud sexual y reproductiva y de la interrupción voluntaria del embarazo. El Título Primero articula los conceptos y medidas (sanitarias y educativas) de la salud sexual y reproductiva. En el artículo 11 se establecía el desarrollo del Plan de Estrategia Nacional de Salud Sexual y Reproductiva (ENSR) que fue publicado por el Ministerio de Sanidad y Política Social en enero de 2011. La ENSR marca entre las recomendaciones de la salud sexual emprender acciones para promover una sexualidad saludable.

Es importante conocer cuáles son los factores que influyen en el comportamiento sexual de los jóvenes. La educación sexual es cada vez más necesaria en unos jóvenes que parecen estar bien informados pero que, sin embargo, presentan altos niveles de riesgo (Failde, et al., 2007; Navarro et al., 2004; Paz et al., 2010).

Los universitarios presentan una elevada aceptación de las relaciones sexuales y la anticoncepción, reflejando el cambio que se originó en España entre los años setenta y ochenta en las actitudes sexuales no maritales (Durex, 2004). Las mujeres vinculan las relaciones sexuales con las amorosas (Maté \& Acarín, 2010), valorando más la fidelidad. Nuestros resultados indican que las mujeres, efectivamente, se muestran en mayor medida de acuerdo con la necesidad de tener una pareja estable para mantener relaciones sexuales. La encuesta nacional también afirmaba que "amar y ser amado" es más importante en las mujeres para mantener relaciones sexuales $(45.5 \%$ de las mujeres y $32 \%$ de los hombres). Meston y Buss (2004) también hallaron que una de las principales razones para tener relaciones sexuales era querer mostrar afecto y expresar amor, aunque los hombres se mostraron más propensos a tener relaciones sexuales ante la ocasión y una persona dispuesta. Sin embargo, Maté y Acarín (2011) informan que en los emparejamientos ocasionales las mujeres valoran la simpatía y la posición social, en tanto los hombres buscan la juventud y el atractivo físico. 
Los hombres, en consonancia con los resultados de otras investigaciones (Muñoz-Silva et al., 2009; Navarro et al., 2004; Teva et al., 2011), presentan una actitud más negativa hacia el empleo de los preservativos.

Respecto al género, los resultados de la doble moral coinciden con las investigaciones previas indicando mayor presencia del machismo en los hombres (Díaz et al., 2010; Sierra et al., 2007), aunque un 10\% de las mujeres se manifiestan conformes con las creencias machistas. A pesar de los cambios sociales de las últimas décadas, el amor romántico está generalizado (Ferrer, et al., 2008a; Schäfer, 2008) también entre los jóvenes universitarios que aceptan mayoritariamente las creencias de los mitos (Esteban \& Tavora, 2008; Ferrer, Bosch \& Navarro, 2010). Los mitos románticos siguen transmitiéndose en el proceso de socialización (Juan, 2011) y en la narrativa escrita y visual (Falcón, 2009), marcando las pautas socioculturales de las relaciones sexuales en pareja. El nivel de aceptación de los celos románticos es similar al informado por Ferrer, Bosch, Navarro y Ferreiro (2010). Supone una reducción sobre los datos de investigaciones anteriores (CIS, 1995; Barrón \& Martínez-Í̃nigo, 2001), pero debemos destacar el peligro que encierra para la justificación de comportamientos de poder y dominación en la pareja. Las situaciones de celos pueden provocar reacciones emocionales violentas ante la percepción de abandono por parte de la pareja (López-Zafra, Rodríguez-Espartal \& Jiménez, 2008).

Nuestros resultados reflejan la influencia del género sobre las actitudes sexuales. El género podría actuar mediando los comportamientos sexuales a través del componente actitudinal. Hemos observado una influencia del género en la actitud sexual de los estudiantes universitarios centrada en el rechazo de los métodos anticonceptivos y, particularmente, de los preservativos. Conforme se incrementan los niveles de machismo y romanticismo, mayor es la tendencia a rechazar las medidas preventivas en los comportamientos sexuales.

Se confirma la influencia del machismo en el comportamiento sexual a través de una actitud negativa (social y personal) hacia el empleo de los preservativos (Caron et al., 1993; Pleck et al., 1993). También se confirma la influencia del romanticismo hipotetizada por Muñoz-Silva et al. (2009) sobre la sexualidad. La interacción del sexo con el romanticismo en el factor de rechazo personal del preservativo alcanza significación en las mujeres. Parece confirmarse que las mujeres cuando tienen relaciones sexuales en el marco de relaciones románticas se perciben seguras y rechazan las medidas preventivas (Calafat et al., 2009; Teva et al., 2009). Otros autores ya habían indicado la influencia del amor romántico sobre las relaciones amorosas (Charkow \& Nelson, 2000; Moreno, González \& Ros, 2007) y su estrecha relación con los comportamientos sexuales, especialmente en el caso de las mujeres (Barrón et al., 1999).

En estudios posteriores sería conveniente completar la medida del machismo con la Escala de Machismo Sexual (Díaz et al., 2010), para analizar con mayor profundidad la vinculación de esta variable con el comportamiento sexual. Por otra parte, el machismo actual es un machismo encubierto. Se manifiesta con comportamientos ocultos e invisibles que han sido denominados micromachismos por Bonino (1995). Se refieren a conductas sutiles y cotidianas que siguen intentando controlar a la mujer, pero que se producen bajo una cubierta de protección legitimada por el entorno social. Esto hace que pase desapercibido, en muchas ocasiones, para las mujeres e incluso que sea aceptado. Teniendo en cuenta esta premisa, también sería adecuado completar el cuestionario con la medición de los micromachismos empleando la categorización más reciente (Bonino, 2005; Ferrer, Bosch, Navarro, Ramis \& García, 2008b) para conocer con mayor certeza los comportamientos sexistas encubiertos.

Consideramos que los resultados son suficientemente interesantes para continuar trabajando en esta línea de investigación y perfilar nuevos estudios a partir de las variables de género. En sucesivas investigaciones es necesario también ampliar la muestra de estudio e incrementar la representación de los hombres para poder obtener una mayor representatividad en los resultados.

Entre las limitaciones del estudio, es importante tener en cuenta que el cuestionario estaba basado en la autodeclaración de los comportamientos sexuales, por lo que la validez de las respuestas es limitada, pudiendo dar lugar a cierto sesgo de información. No obstante, el anonimato de los cuestionarios favorece una mayor sinceridad. En segundo lugar, este estudio forma parte de una investigación más amplia, por lo que sólo se exponen algunos datos. Por último, el carácter transversal del estudio nos impide establecer una relación causal clara.

A pesar de las limitaciones comentadas, los resultados reflejan la intervención de la construcción del género en la sexualidad. Los indicios obtenidos demuestran la relevancia de continuar investigando el género, ampliando el conocimiento existente sobre su 
relación con el comportamiento sexual y las actitudes sexuales, para la formulación de programas preventivos, la intervención y la formación de los jóvenes en salud sexual. Los resultados sugieren que es necesario seguir trabajando para desmontar las creencias de género erróneas y, en particular, el sexismo en cualquiera de sus manifestaciones, concretadas en nuestro estudio a través del machismo y de los mitos románticos.

\section{Referencias}

Barrón, A., Martínez-Íñigo, D., de Paul, P. y Yela, C. (1999). Romantic beliefs and myths in Spain. The Spanish Journal of Psychology, 2(1), 64-73.

Barrón, A. y Martínez-Íñigo, D. (2001). Los celos: una perspectiva psicosocial. Madrid: Aljibe.

Bonino, L. (1995). Desvelando los micromachismos en la vida conyugal. En J. Corsi (Ed.), Violencia masculina en la pareja. Una aproximación al diagnóstico y a los modelos de intervención (pp. 191-208). Buenos Aires: Paidós.

Bonino, L. (2005). Las microviolencias y sus efectos: claves para su detección. En C. Ruiz-Jarabo y P. Blanco (Coords.), La violencia contra las mujeres: prevención y detección (pp. 83-102). Madrid: Díaz de Santos.

Bosch, E. y Ferrer, V.A. (2002). La voz de las invisibles. Las víctimas de un mal amor que mata. Madrid: Cátedra.

Calafat, A., Juan, M., Becoña, E., Mantecón, A. y Ramón, A. (2009). Sexualidad de riesgo y consumo de drogas en el contexto recreativo. Una perspectiva de género. Psicothema, 21(2), 227-233.

Caron, S.L., Davis, C.M., Halteman, W.A. y Stickle, M. (1993). Predictors of condom-related behaviours among first-year students. Journal of Sex Research, 30, 252-259.

Castañeda, M. (2002). El machismo invisible. México: Grijalbo.

Chaplin, J.R. (2000). Adolescent sex and mass media: A developmental approach. Adolescence, 35, 799-811.

Charkow, W. y Nelson, E. (2000). Relationship dependency, dating violence and scripts of female. Journal of College Counselling, $3(1), 12-17$.

CIS (1995). Actitudes y conductas afectivas de los españoles. Datos de Opinión, 7. Recuperado de www.cis.es

De Lemus, S., Moya, M. y Glick, P. (2010). When contact correlates with prejudice: Adolescents' romantic relationship experience predicts greater benevolent sexism in boys and hostile sexism in girls. Sex Roles, 63, 214-225.

Díaz, C.L., Rosas, M.A. y González, M.T. (2010). Escala de Machismo Sexual (EMS-Sexismo-12): diseño y análisis de propiedades psicométricas. SUMMA Psicológica UST, 7(2), 35-44.

Diéguez, K.L., Sueiro, E. y López, F. (2003). The Sexual Double Standard y variables relacionadas. Cuadernos de Medicina Psicosomática y Psiquiatría de Enlace, 67/68, 79-88.

Durex (2004). Durex global sex survey. Recuperado de http://www2. hu-berlin.de/sexology/ECE6/html/gss2004result.pdf

Esteban, M.L. y Tavora, A. (2008). El amor romántico y la subordinación social de las mujeres: revisiones y propuestas. Anuario de Psicología, 39(1), 59-73.

Failde, J.M., Lameiras, M. y Bimbela, J.L. (2008). Prácticas sexuales de chicos y chicas españoles de 14-24 años de edad. Gaceta Sanitaria, 22(6), 511-519.

Falcón, L. (2009). ¿Cómo tengo que ser para que me quieras? La construcción del enamoramiento en los relatos cinematográficos: propuesta de un modelo de alfabetización audiovisual para la prevención de la violencia de género. Revista de Estudios de Juventud, 86, 65-81.

Ferrer, V.A., Bosch, E., Navarro, C. y Ferreiro, V. (2010). El mito romántico de los celos y su aceptación en la sociedad española actual. Apuntes de Psicología, 28(3), 391-402.

Ferrer, V.A., Bosch, E., Navarro, C., Ramis, M.C. y García, M.E. (2008a). El concepto de amor en España. Psicothema, 20(4), 589-595.

Ferrer, V.A., Bosch, E., Navarro, C., Ramis, M.C., y García, M.E. (2008b). Los micromachismos o microviolencias en la relación de pareja: Una aproximación empírica. Anales de Psicología, 24(2), 341-352.

Ferrer, V.V., Bosch, E. y Navarro, C. (2010). Los mitos románticos en España. Boletín de Psicología, 99, 7-31.

García-Vega, E., Menéndez, E., García, P. y Rico, R. (2010). Influencia del sexo y del género en el comportamiento sexual de una población adolescente. Psicothema, 22(4), 606-612.

Giddens, A. (1995). La transformación de la intimidad. Sexualidad, amor y erotismo en las sociedades modernas. Madrid: Gráficas Rógar.

INJUVE (2008). Informe Juventud en España. Recuperado de http:// www.injuve.es

Juan, M.L. (2011). El amor romántico es una construcción cultural y social. Agathos: Atención Sociosanitaria y Bienestar, 2, 58-61.

López-Zafra, E., Rodríguez-Espartal, N. y Jiménez, M.I. (2008). Cultura de honor e inteligencia emocional: ¿conceptos relacionados o incompatibles? SUMMA Psicológica UST, 5(2), 17-26.

Maté, C. y Acarín, N. (2010). Las relaciones sexuales, encuesta a los estudiantes de la Universitat Pompeu Fabra, Barcelona (20 a 27 años). SUMMA Psicológica UST, 7(2), 93-108.

Maté, C. y Acarín, N. (2011). Encuesta sobre la elección de pareja a estudiantes de la Universitat Pompeu Fabra, Barcelona (20 a 27 años). SUMMA Psicológica UST, 8(1), 37-46.

Medrano, A. (2012). Desigualdades de género. Bilbao: Mensajero.

Meston, C. y Buss, D.M. (2007). Why humans have sex. Archives of Sexual Bahavior, 36, 477-507.

Milhausen, R.R. y Herold, E.S. (1999). Does the sexual double standard still exist? Perceptions of university women. The Journal of Sex Research, 36, 361-368.

Ministerio de Sanidad y Política Social (2009). Resultados de la encuesta nacional de salud sexual 2009. Recuperado de http:// www.mujeressaludygenero.com/contenido/v5_presentacion ResultadosENSS_16dic09.pdf

Moreno, M. (2003). Sumisión aprendida: Un estudio sobre la violencia de género. Anuario de Psicología, 34(2), 235-251.

Moreno, M., González, A. y Ros, M. (2007). Enamoramiento y violencia contra las mujeres. En V.A. Ferrer y E. Bosch (Comps.), Los feminismos como herramientas de cambio social (II): de la violencia contra las mujeres a la construcción del pensamiento feminista (pp. 21-34). Palma: UIB.

Muñoz-Silva, A., Sánchez-García, M., Martius, A. y Nunes, C. (2009). Gender differences in HIV-Related sexual behaviour among college students from Spain and Portugal. The Spanish Journal of Psychology, 12(2), 485-495.

Navarro, Y., Carrasco, A. M., Sánchez, J.C. y Torrico, E. (2004). Comportamiento y actitudes sexuales en adolescentes y jóvenes. Archivos Hispanoamericanos de Sexología, 10(2), 167-182.

Organización Mundial de la Salud (2006). Gender and reproductive rights. Glossary. Recuperado de http://www.who.int/reproductivehealth/gender/glossary.html

Paz, M., Castro, A., Madrid, J. y Buela-Casal, G. (2010). Análisis de la conducta sexual de adolescentes autóctonos e inmigrantes 
latinoamericanos en España. International Journal of Clinical and Health Psychology, 10(1), 89-103.

Petersen, J.L. y Hyde, J.S. (2010). A meta-analytic review of research on gender differences in sexuality. Psychological Bulletin, 136(1), 21-38.

Pleck, J.H., Sonenstein, F.L. y Ku, L.C. (1993). Masculinity ideology: Its impact on adolescent males heterosexual relationships. Journal of Social Issues, 49(3), 11-29.

Puente, D., Zabaleta, E., Rodríguez-Blanco, T., Cabanas, M., Monteagudo, M., Pueyo, M.J., Jané, M. Mestre, N., Mercader, M. y Bolíbar, B. (2011). Gender differences in sexual risk behaviour among adolescents in Catalonia, Spain. Gaceta Sanitaria, 25(1), 13-19.

Schäfer, G. (2008). Romantic love in heterosexual relationships: women's experiences. Journal of Social Sciences, 16(3), 187-197.

Selva, M. (2011). Plan de políticas de mujeres 2008-2011. En F. Esteve y M. Illamola (Coords.), La igualdad por razón de sexo en la Unión Europea (pp. 257-259). Gerona: Servei de Publicacions Universitat de Girona.

Sierra, J.C., Rojas, A., Ortega, V. y Marín, J.D. (2007). Evaluación de actitudes sexuales machistas en universitarios: primeros datos psicométricos de las versiones españolas de la Double Standard Scale (DSS) y de la Rape Supportive Attitude Scale (RSAS). International Journal of Psychology and Psychological Therapy, 7(1), 41-60.

Teva, I., Paz, M. y Buela-Casal, G. (2009). Characteristics of sexual behaviour in Spanish adolescents. The Spanish Journal of Psychology, 12(2), 471-484.

Teva, I., Paz, M. y Buela-Casal, G. (2011). Búsqueda de sensaciones sexuales, estilos de afrontamiento, estrés social y su relación con la conducta sexual adolescente. Anales de Psicología, 27(1), 35-46.

Ubillos, S., Zubieta, E., Páez, D., Deschamps, J.C., Ezeiza, A. y Vera, A. (2001). Amor, cultura y sexo. Revista Electrónica de Motivación y Emoción (REME), 4(8-9). Recuperado de http:// reme.uji.es/articulos/aubils9251701102/texto.html.

Yela, C. (2003). La otra cara del amor: mitos, paradojas y problemas. Encuentros en Psicología Social, 1(2), 263-267.

Yubero, M., Yubero, S. y Larrañaga, E. (en prensa). Actitudes y comportamiento sexual de riesgo de embarazo en jóvenes universitarios. Journal of Child and Adolescents Psychology. 
